\title{
Un método alternativo para fijar drenajes
}

\section{An alternative way to secure drains}

\author{
Solesio Pilarte, F.*, Laredo Ortiz, C.*, Lorda Barraguer, E.**
}

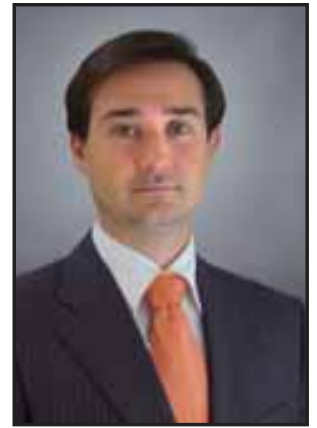

Solesio Pilarte, F.

\section{Resumen}

La colocación de drenajes en las intervenciones quirúrgicas es una práctica muy habitual en la mayoría de las operaciones de Cirugía Plástica y también de otras especialidades, pero en ocasiones su objetivo fracasa al deslizarse el drenaje. Diversos autores han propuesto varios métodos de sujeción de los drenajes a la piel con distinto grado de aceptación. El más empleado es el método de "sandalia romana" que, si no se realiza correctamente resulta ineficaz.

En el presente artículo analizamos dónde y por qué falla frecuentemente dicho método y proponemos un procedimiento alternativo, sencillo y seguro para la fijación de drenajes quirúrgicos, el uso del ballestrinque.

\section{Palabras clave Drenajes quirúrgicos.}

Código numérico 14-15815
Key words Surgical drains.

Numeral Code $\quad 14-15815$ 


\section{Introducción}

La historia de los nudos es más antigua que la de las cuerdas y tal vez más que la especie humana. Incluso en el reino animal se tiene noticia de la elaboración de nudos. Cuando aún no existían cuerdas se empleaban fibras vegetales para, atándolas, construir herramientas o simplemente agrupar objetos. Desde que el hombre tuvo la necesidad de utilizar cuerdas también tuvo la necesidad de sujetar objetos y con ello de utilizar nudos. En Medicina, se tiene constancia del empleo de nudos por lo menos desde el siglo primero de nuestra era, gracias a los escritos de Heraklas (1), un médico griego que describió 16 nudos empleados en la Medicina de la época.

El empleo de los drenajes se remonta a la época de Hipócrates, que describió el empleo de las cánulas. En 1895 Kellog describió el precursor del drenaje aspirativo. Tres años después Heaton aplicó aspiración constante a un drenaje en sifón (2).

Drenajes y nudos forman un tándem inseparable en nuestra cirugía mayor y, en ocasiones, también en cirugía menor. Son varios los autores (3-7) que han propuesto diversas formas de fijar los drenajes, la mayoría atando el drenaje directamente, aunque se ha propuesto también un método consistente en adherir un Steri Strip ${ }^{\circledR}$ de papel al drenaje y suturar el mismo a la piel (8). Dini (9) propone, a diferencia del resto de métodos, fijar el drenaje por su extremo más alejado de la botella.

En cualquier caso, el método más extendido es el denominado en "sandalia romana", que podríamos decir que consiste en fijar un punto (habitualmente de seda) a la piel y con sus dos extremos libres ir rodeando el drenaje en direcciones opuestas, cruzándose por delante y por detrás del mismo (Fig. 1 y 2).

Al realizar la fijación con este método la mayoría de las lazadas cruzan en oblicuo el tubo de drenaje, a excepción de la última, que es la que realmente sujeta el drenaje al ser perpendicular. Recomendamos ver el dibujo de la Figura 3 para entender bien este punto.

Como se puede apreciar, la circunferencia de la sección del drenaje es de menor longitud que las espirales oblicuas que lo sujetan. Si la última vuelta de la sutura no está muy apretada, esta resbalará, acercándose todas las vueltas hacia la superficie cutánea como si comprimiésemos un muelle, y al suceder esto, las vueltas oblicuas de la sutura se horizontalizan liberando el drenaje (Fig. 4).

En ocasiones, el drenaje puede salirse de su ubicación original aun permaneciendo el nudo inmóvil; esto sucede cuando existe una gran distancia entre los puntos de anclaje a la piel y al drenaje (puntos A, B y
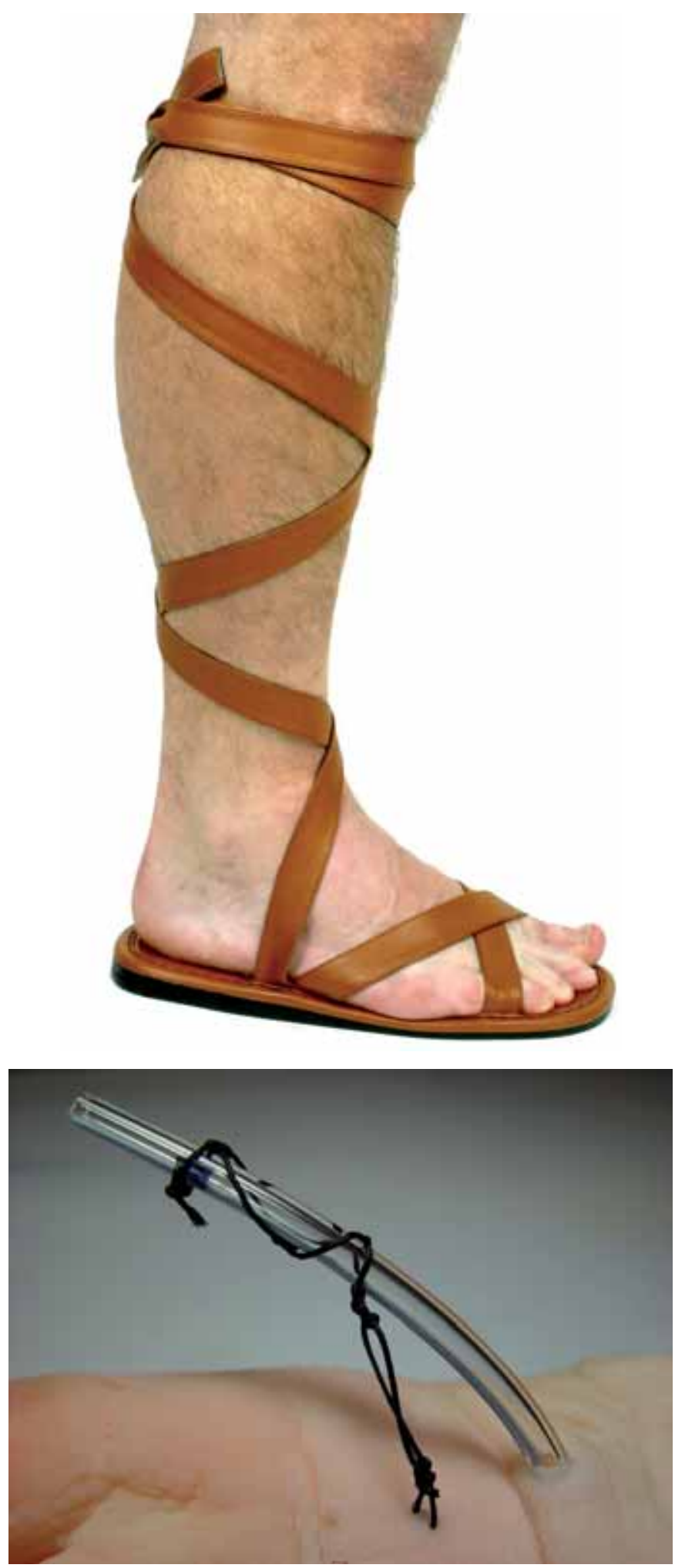

Fig. 1 y 2. Sandalia romana y drenaje sujeto de modo similar.

se tiene por costumbre dejar una lazada generosa al comienzo del anudado, justo antes de comenzar a rodear el drenaje. Cuando esto sucede, aunque la distancia entre los puntos A y B sea constante, y gracias a la flexibilidad del drenaje, se puede formar un seno que permita la extracción del mismo (Fig.5). Por todo esto, hemos querido adaptar un nudo conocido desde la antigüedad y descrito por Heraklas(1) para fijar con mayor efectividad los drenajes. Dicho nudo, también 

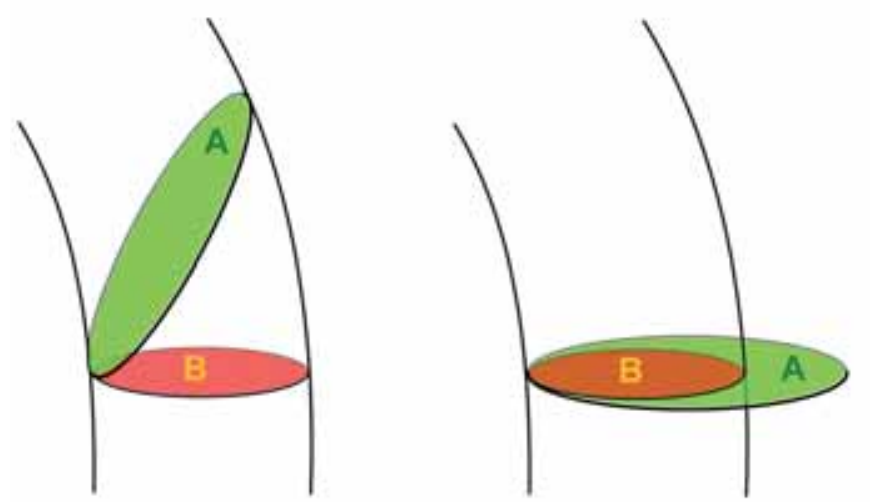

Fig. 3. La sección oblicua del drenaje es mayor que la perpendicular.

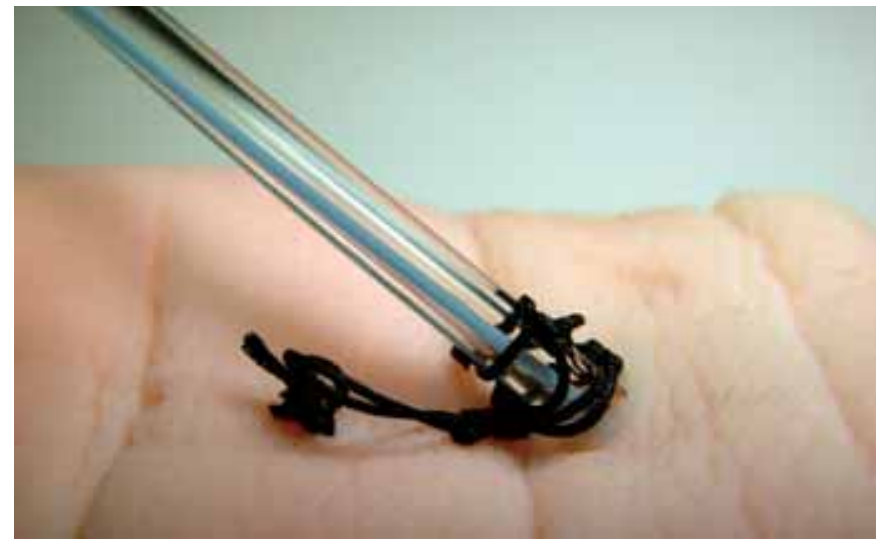

Fig. 4. Si el drenaje deja de traccionar de la seda, ésta se colapsa en acordeón liberando el drenaje.

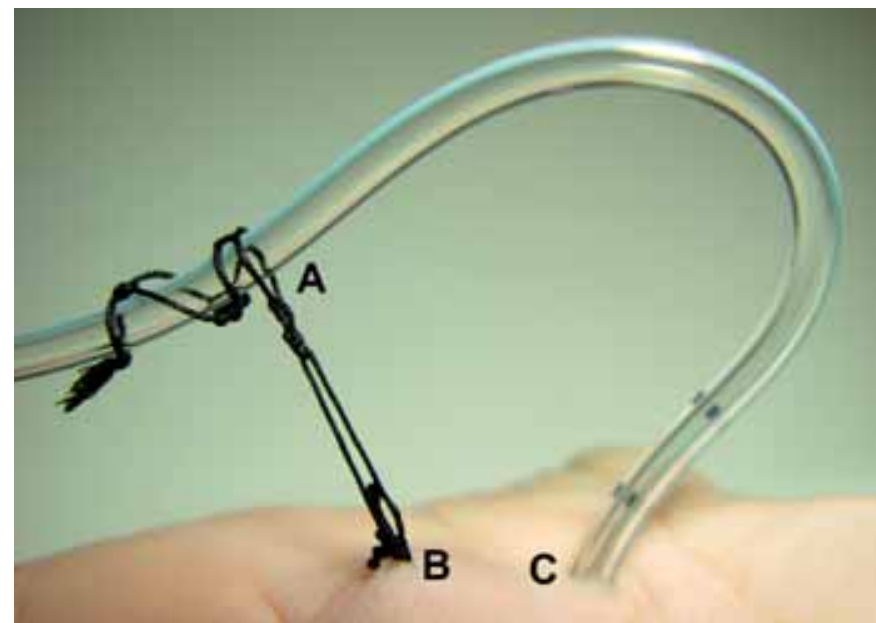

Fig. 5. Si los puntos A, B y C no están próximos, corremos el riesgo de que se salga el drenaje aun siendo firme el nudo.

conocido como "ballestrinque" es usado desde hace siglos por los marineros, ya que, entre sus características destaca el ser ideal para la sujeción de elementos cilíndricos. Nuestra afición al mar nos hizo ver su gran utilidad.

\section{Material y método}

Proponemos el empleo de un nudo marinero, el ballestrinque, no empleado en la práctica quirúrgica, para fijar los drenajes. Dicho nudo es efectivo para
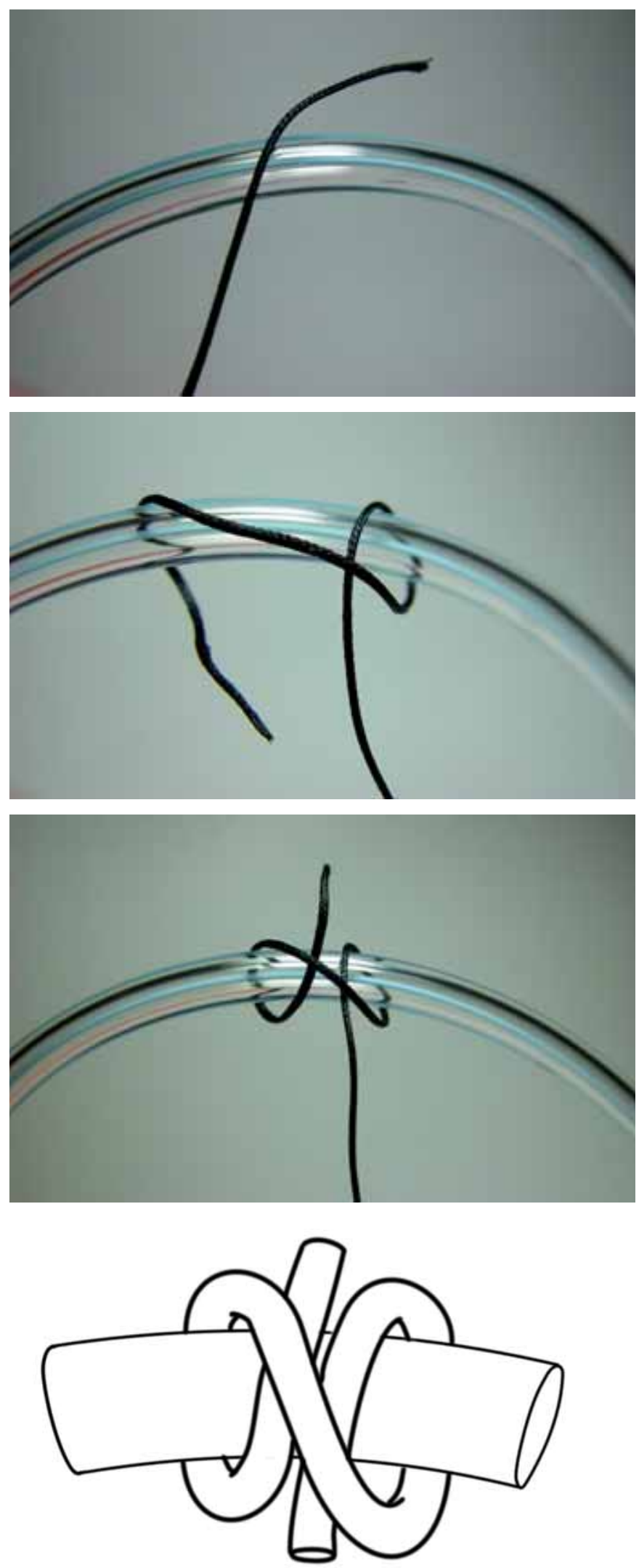

Fig. 6 a, b y c. Pasos del ballestrinque. Figura 6 d: Ballestrinque terminado.

sujetar firmemente un objeto tubular (en nuestro caso el drenaje) y por hacerse más firme si se realiza una tracción del mismo. En el presente artículo explicamos cómo realizar dicho nudo y algunos consejos prácticos para que su uso resulte más sencillo. 
Para la ejecución de este método de fijación de drenajes, aconsejamos colocar primero el drenaje, luego anudar la seda al drenaje y por último dar el punto a la piel. Veamos los pasos para la realización del ballestrinque:

$1^{\circ} \mathrm{La}$ sutura rodea al drenaje pasando primero por encima del mismo y completando la vuelta por debajo (Fig. $6 \mathrm{~A})$.

$2^{\circ}$ A continuación, siguiendo la misma dirección de giro, realizamos otra vuelta por encima de la primera (Fig. 6 B).

$3^{\circ}$ Por último, pasamos el extremo libre de la sutura por debajo de esta última y sin cruzar la primera. Ya está hecho el nudo (Fig. 6 C, D).

$4^{\circ}$ Seguidamente sólo queda ajustarlo al drenaje y dar un punto a la piel.

Es importante que el ballestrinque quede cercano a la piel, ya que si no podría salirse el drenaje tal y como se ha descrito previamente. Aconsejamos una distancia menor de $1 \mathrm{~cm}$.

Todos sabemos que la realización de nudos es más complicada usando guantes. Por ello hemos querido proponer una maniobra alternativa para la realización del nudo (Fig. 7):

$1^{\circ}$ Abrimos la palma de la mano izquierda y pronosupinamos hasta que el pulgar apunte a nuestra cara.

Fig. 7. Método alternativo para anudar un ballestrinque.
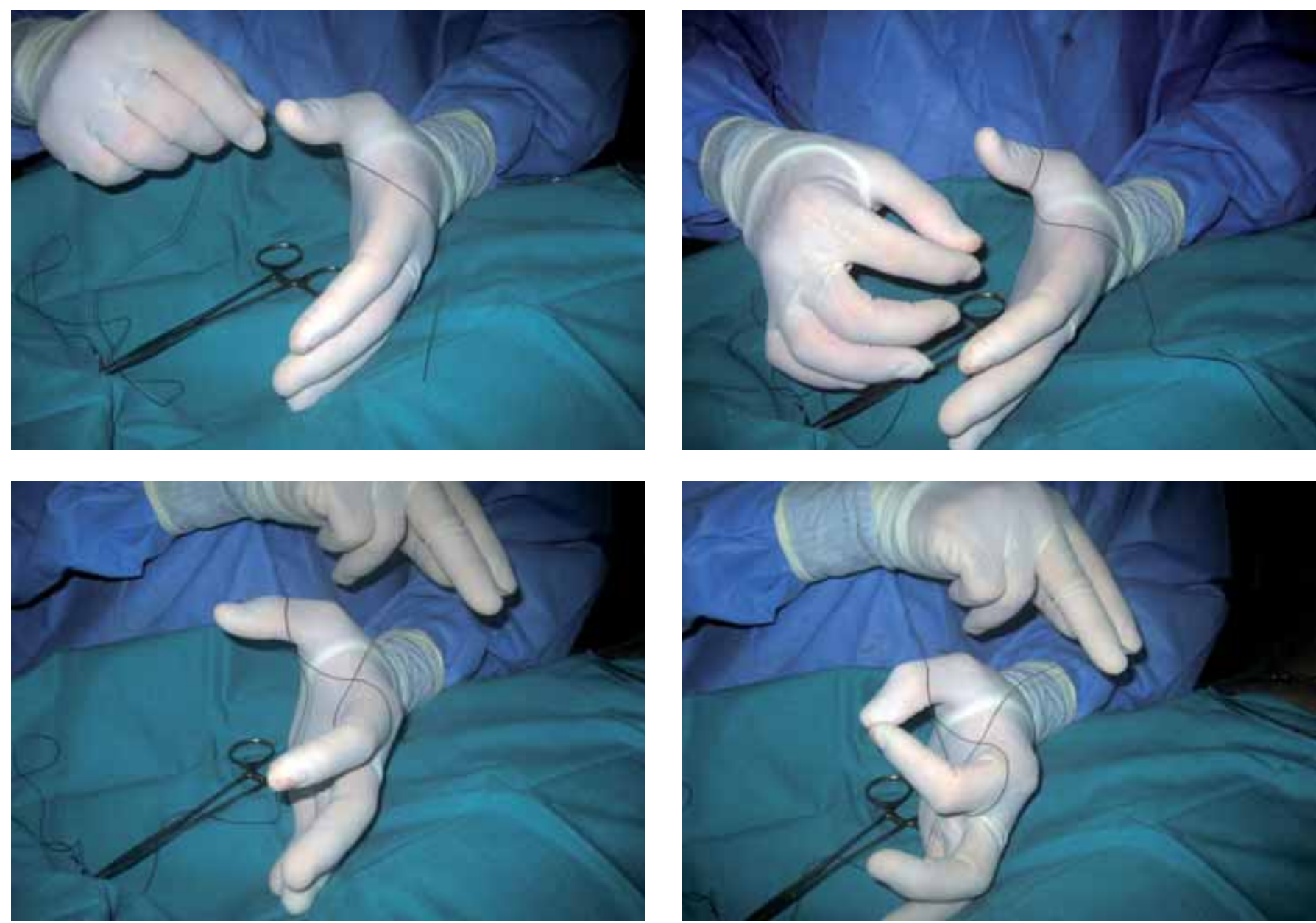

$2^{\circ}$ Dejamos caer la sutura en la primera comisura, quedando a un lado la aguja y al otro el extremo libre de la seda.

$3^{\circ}$ Con uno de los extremos rodeamos el pulgar pasando por el dorso del primer metacarpiano y seguidamente por la primera comisura.

$4^{\circ}$ Con el otro extremo rodeamos el dedo índice pasando por la segunda comisura y después por la primera. Habremos conseguido una figura con forma de 8.

$5^{\circ}$ A continuación juntamos los pulpejos del pulgar $y$ del índice.

$6^{\circ}$ Desplazando la lazada que rodea el índice hasta juntarla con la del pulgar obtendremos un ballestrinque rodea nuestro pulgar.

$7^{\circ}$ Con cuidado sujetamos todo el nudo en bloque e introducimos en su interior el drenaje

$8^{\circ}$ Solo queda fijarlo a la piel como se ha descrito previamente.

\section{Resultados}

Hemos empleado el método propuesto para la fijación de drenajes durante los últimos 6 años, periodo durante el cual no hemos observado la pérdida de 

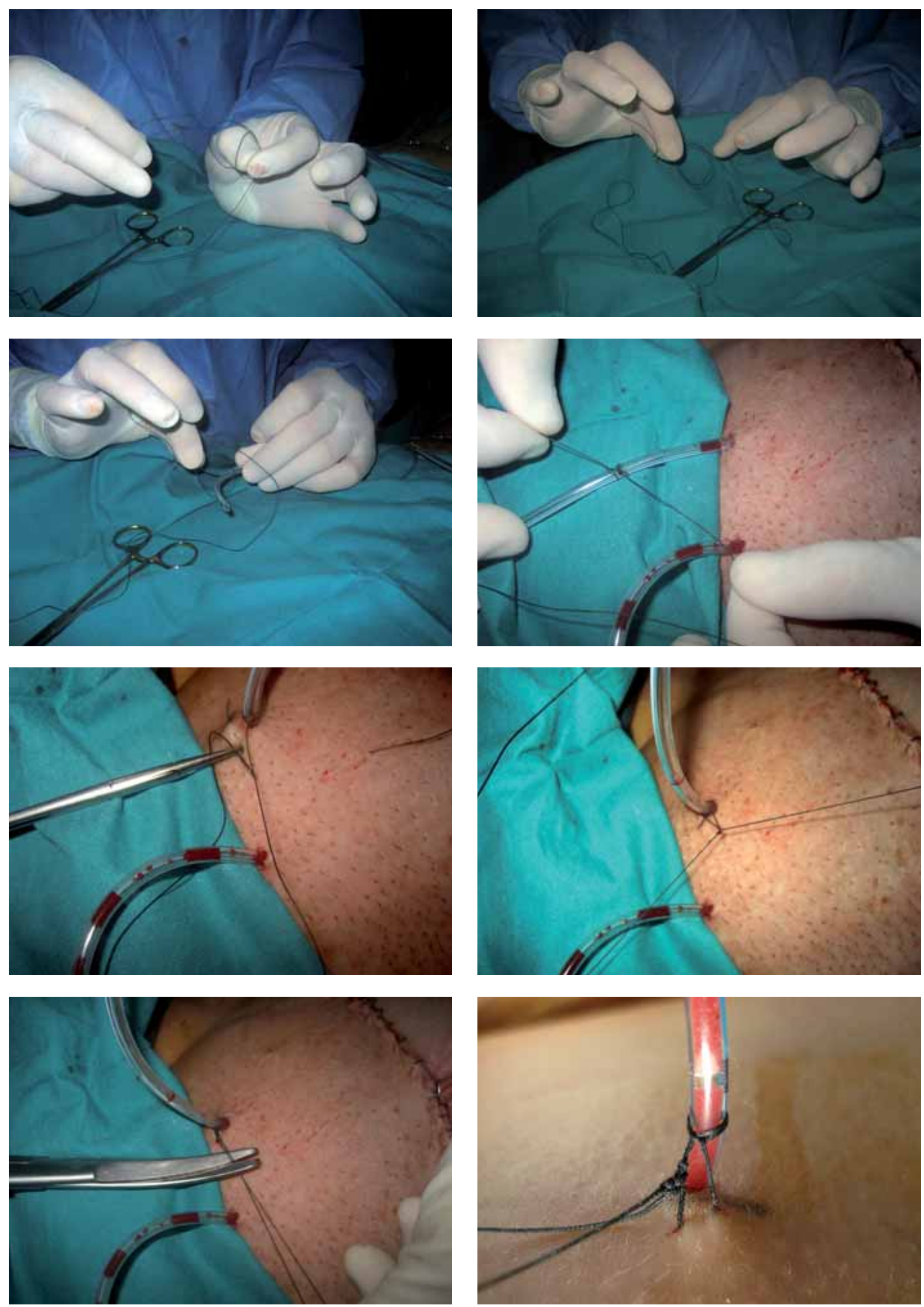
vacío por deslizamiento de ningún drenaje de Redón. Podemos afirmar por tanto, que es un método efectivo y fiable.

\section{Discusión}

Además de sus usos náuticos, el ballestrinque se usa, por ejemplo, para sujetar colgados los jamones; la sujeción de los mismos mediante un nudo no es sencilla, ya que es un elemento rígido en el que no se puede hender la cuerda, es pingüe y pesado, motivos por los cuales la sujeción debe ser firme. La elección del nudo no es casual, sino consecuente a su efectividad en situaciones como la descrita.

Pocos son los artículos que realizan un estudio analítico comparando distintos tipos de nudos. El ballestrinque es un nudo muy útil para el propósito descrito en este trabajo. Lovett (10) comparó en 2006 la eficacia de sistemas comerciales para la fijación de tubos endotraqueales frente a distintos tipos de nudos. En sus resultados, el ballestrinque resultó ser más eficaz, aunque la diferencia no fue estadísticamente significativa, lo cual es lógico teniendo en cuenta el escaso tamaño de la muestra.

En nuestra propia experiencia el ballestrinque ha demostrado ser de utilidad, aunque el presente artículo no tiene como finalidad demostrar su eficacia mediante el empleo de estadística al no ser el nuestro un trabajo analítico, sino puramente descriptivo. Nuestra intención es solo el presentar un método que creemos útil para que pueda ser compartido por otros profesionales.

\section{Conclusiones}

El empleo de drenajes es algo muy habitual en nuestras cirugías. Su fijación puede ser mejorada anudando el drenaje con un nudo marinero llamado ballestrinque y situando éste cerca de la piel. De este modo evitamos deslizamientos del drenaje, disminuyendo el riesgo de complicaciones. Proponemos pues el uso del ballestrinque como sistema de fijación de drenajes quirúrgicos.

\section{Dirección del autor}

Dr. Francisco Solesio Pilarte

Hospital General Universitario de Alicante.

C/ Pintor Baeza 12. (03010). Alicante. España

e-mail: solesio_fra@gva.es

\section{Bibliografía}

1. Hage, J. J..: "Heraklas on Knots: Sixteen Surgical Nooses and Knots from the First Century A.D” World Journal of Surgery, 2008: Volume 32, Number 4.

2. López K., F.: "Uso de drenajes en cirugía". http://www.cirugest.com/htm/revisiones/cir01-05/01-05-01.htm.

3. Lim M, Pitkin L, Spraggs P.: "A new head and neck surgical drain fixation technique.” Journal of Laryngology \& Otology. 2005, 119 (5): 396 .

4. Frank, M.: "A simple technique for securing tubes". Annals of Emergency Medicine.1983, 12(1): 25.

5. Wigle, RL.: "A simple technique for securing tubes and drains". Surg Gynecol Obstet 1992, 174(3):232.

6. Jandhyala R., Healy C.: "A new technique for securing drains to the scalp". Annals of the Royal College of Surgeons of England, 2007, 89 (7):726.

7. Leong, S., Mortimore, S.: "Securing head and neck surgical drains made easy". Clinical Otolaryngology, 2006. 31 (5): 467.

8. Bellioni, M.D., delli Santi G., y Loreti A.: "Flag drain fixation: a secure method". Plast And Rec. Surg. 2005, 115 (6):1805.

9. Dini GM, de Castilho HT, Ferreira LM.: "A simple technique to ensure drain fixation". Plast Reconstr Surg. 2003, 112 (3): 923.

10. Lovett P., Flaxman A., Stürmann K., Bijur P.: "The insecure airway: a comparison of knots and commercial devices for securing endotracheal tubes". BMC Emergency Medicine 2006., Volume 6:7. http://www.biomedcentral.com/bmcemergmed 